01

\title{
Эволюция пространственно-временного хаоса в дискретно-непрерывной активной среде
}

\author{
() В.А. Максименко ${ }^{1}$, Д.Э. Постнов ${ }^{2}$, А.А. Короновский ${ }^{2}$, \\ B.B. Макаров ${ }^{1}$, A.E. Храмов ${ }^{1}$ \\ ${ }^{1}$ Саратовский государственный технический университет \\ им. Гагарина Ю.А., Саратов \\ ${ }^{2}$ Саратовский государственный университет им. Н.Г. Чернышевского, \\ Саратов \\ E-mail: maximenkovl@gmail.com
}

Поступило в Редакцию 28 апреля 2017 г.

Для диагностики степени регулярности волновых структур в модельной нейросистеме предложен и применен специальный подход к расчету спектра показателей Ляпунова. Продемонстрирован и охарактеризован количественно переход между режимами регулярной волновой динамики и развитого пространственно-временного хаоса.

DOI: $10.21883 /$ PJTF.2017.12.44714.16443

Развитие знаний о функционировании мозга заставляет периодически пересматривать парадигмы физических моделей нейросистем. В частности, современные представления о совокупности процессов, сопровождающих такие явления, как распространяющаяся кортикальная депрессия, мигрень, волны деполяризации при мозговых травмах или инсульте, выходят далеко за рамки модельного представления ансамбля нейронов как дискретного набора активных элементов с той или иной топологией межэлементных связей [1-4]. В данных случаях указанный дискретный набор элементов оказывается „встроен“ в диффузионную среду, которая играет роль аккумулятора и проводника веществ, управляющих активностью нейронов. При этом адекватные поставленной задаче математические модели принадлежат к классу дискретнонепрерывных систем, исследование динамики которых зачастую требует специального подхода. На максимально упрощенном уровне такая нейросистема может быть описана с помощью трехкомпонентной модели 
типа реакция-диффузия, в которой уравнение в частных производных описывает распространения веществ (ионы калия, глутамат калия) в межклеточном пространстве, в то время как набор обыкновенных дифференциальных уравнений (ОДУ) определен в дискретном наборе позиций и характеризует активность популяции нейронов [5].

Целью данной работы является количественный анализ обнаруженного при численном моделировании эффекта перехода от режима пространственно-временного хаоса к регулярному режиму бегущих волн в модельной системе, описывающей дискретную популяцию нейронов ФитцХью-Нагумо [6], размещенных в узлах двумерной пространственной сетки и взаимодействующих друг с другом посредством диффузионной связи по дополнительной переменной, непрерывно распределенной в пространстве взаимодействия.

Рассмотрим исследуемую математическую модель. В предположении, что нейроны располагаются в точках с координатами $r_{i j}=\left(x_{i}, y_{j}\right)$, где $x_{i}=i h x, y_{j}=j h y$, a $h$ - пространственный шаг, их динамика может быть описана при помощи набора ОДУ следующим образом:

$$
\begin{aligned}
& \varepsilon_{v} \frac{d v_{i j}(t)}{d t}=v_{i j}(t)-v_{i j}^{3}(t) / 3-w_{i j}(t)+z\left(x_{i}, y_{j}, t\right), \\
& \tau_{l} \frac{d w_{i j}(t)}{d t}=A+B v_{i j}(t)-w_{i j}(t),
\end{aligned}
$$

где $v_{i j}(t)$ и $w_{i j}(t)$ - переменные, характеризующие электрическую активность каждого нейрона, $i, j=\overline{1,40}$. В свою очередь, функция $z\left(x_{i}, y_{j}, t\right)$, определенная непрерывно на пространстве $\mathbf{r}=(x, y)$, описывает пространственно-временную эволюцию концентрации веществ в межклеточном пространстве [5]

$$
\begin{aligned}
& \varepsilon_{z} \frac{\partial z}{\partial t}=\alpha_{z} \Psi(v)-z+\gamma\left(\frac{\partial^{2} z}{\partial x^{2}}+\frac{\partial^{2} z}{\partial y^{2}}\right), \\
& \Psi(v)=\frac{1}{2}\left(1+\tanh \left(\frac{v}{v_{s}}\right)\right) .
\end{aligned}
$$

В уравнении (2) $\Psi(v)$ представляет собой логистическую функцию, зависящую от значения $v$ и имеющую два асимптотических предела - ноль и единицу. Уравнения (1) и (2) дополняются граничными

7 Письма в ЖТФ, 2017, том 43, вып. 12 
условиями, причем граничные условия для переменной $z$ определяются особенностями задачи. В данной работе исследуемая активная среда моделировалась прямоугольным пространством размера $L_{x} \times L_{y}$ $\left(L_{x}=1, L_{y}=1\right)$ со смешанными граничными условиями. В частности, граница для координаты $y$ описывалась граничными условиями Неймана и Дирихле, граничные условия для координаты $x$ были периодическими:

$$
\begin{aligned}
& \varepsilon_{z} \frac{d z_{1, j}}{d t}=\alpha_{z} \Psi\left(v_{1, j}\right)-z_{1, j}+\gamma\left(z_{2, j}-z_{1, j}\right)+k_{d} \gamma\left(z_{N, j}-z_{1, j}\right) \\
& \varepsilon_{z} \frac{d z_{N, j}}{d t}=\alpha_{z} \Psi\left(v_{N, j}\right)-z_{N, j}+\gamma\left(z_{N-1, j}-z_{N, j}\right)+k_{d} \gamma\left(z_{1, j}-z_{N, j}\right),
\end{aligned}
$$

где $z_{1, j}$ и $z_{N, j}, \forall j=1 \ldots N$ соответствуют значениям переменной $z$ на левой и правой границах среды соответственно. Согласно [5], такая конфигурация пространства позволяет при определенных условиях наблюдать возникновение автономного ведущего центра.

Уравнения модели (1)-(3) интегрировались численно с шагом пространственной сетки $h=0.025$. При этом значения параметров системы были заданы как $A=0.5, B=1.1, \tau_{l}=1.0, \varepsilon_{z}=1.0, \alpha_{z}=1.1$, $\varepsilon_{v}=0.004, \gamma=7.5 \cdot 10^{-4}, v_{s}=0.05$. В качестве управляющего параметра использовалась величина $k_{d}$, определяющая конфигурацию пространства. В предельном случае, когда $k_{d}=1$, левая и правая границы оказываются связанными, и между ними возможен диффузионный перенос вещества. В случае, когда $k_{d}=0$, границы оказываются разомкнутыми. В промежуточных случаях $0<k_{d}<1$ является поправкой к коэффициенту диффузии, описывающей перенос вещества между левой и правой гранями.

На рис. 1 представлены результаты численного моделирования динамики системы для предельных случаев $k_{d}=1$ (рис. 1,a) и $k_{d}=0$ (рис. $\left.1, b\right)$ в виде „моментального снимка“ распределений концентрации вещества $z(x, y)$ в межклеточном пространстве. Видно, что в случае, когда $k_{d}=1$ (граница области замкнута), в системе наблюдается регулярная структура, состоящая из волн возбуждения, распространяющихся в одном направлении с одинаковыми скоростями. В случае, когда коэффициент $k_{d}$ становится равным нулю (что соответствует разомкнутой границе), в системе возникает нерегулярная динамика, которой соответствует наличие пространственной области хаотической динамики (ведущего центра), 

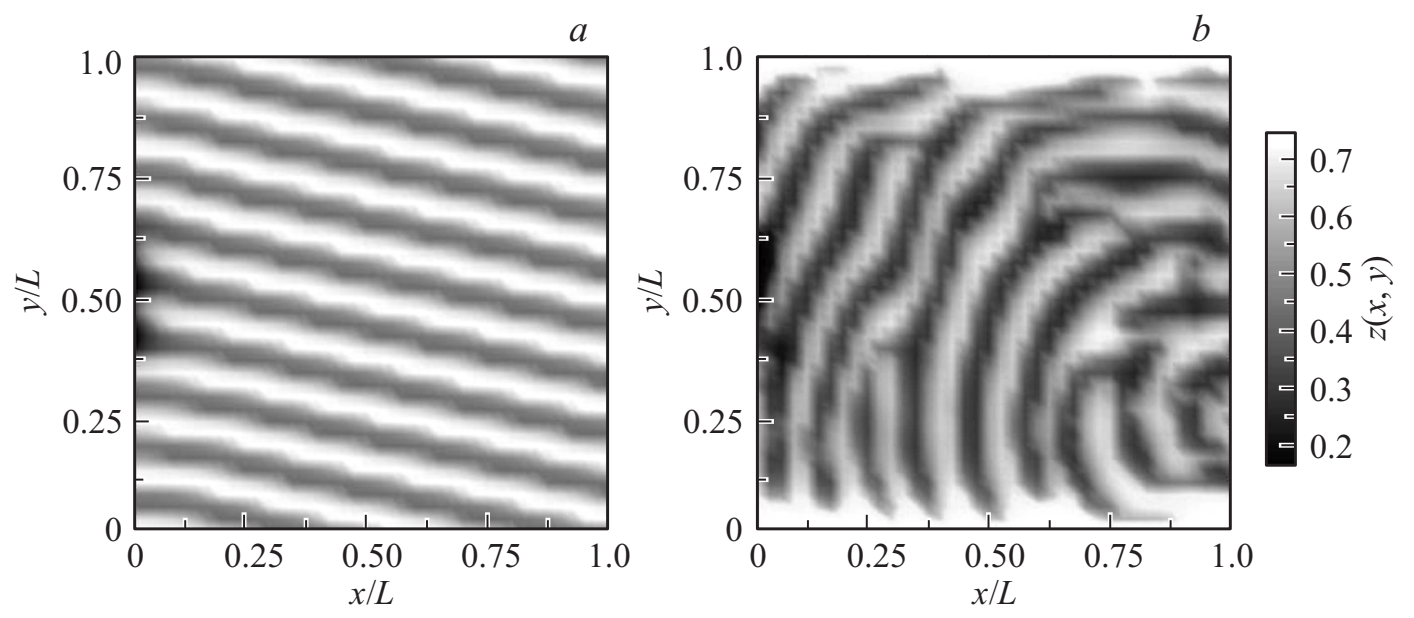

Рис. 1. Моментальные распределения концентрации вещества $z(x, y)$ в межклеточном пространстве, построенные для предельных случаев: $a-k_{d}=1$ (граница замкнута), $b-k_{d}=0$ (граница разомкнута). 

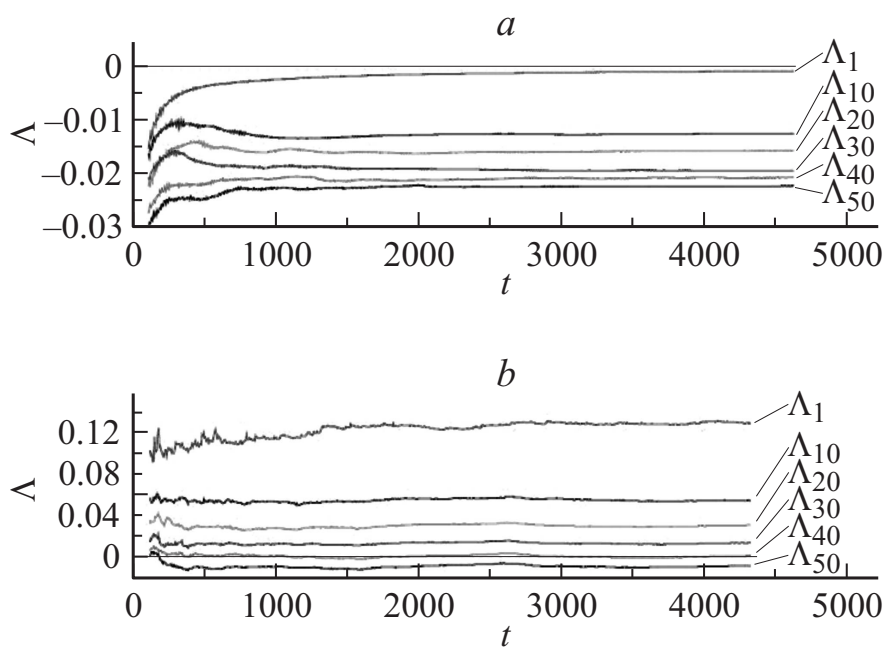

Рис. 2. Зависимости от времени значений нескольких старших показателей Ляпунова для предельных случаев $k_{d}=1(a)$ и $k_{d}=0(b)$.

порождающего набор волн, распространяющихся в разные стороны. Очевидно, что монотонное изменение параметра $k_{d}$ от единицы до нуля обеспечивает переход от периодической динамики к хаотической.

Для количественной характеристики эволюции пространственновременной динамики в настоящей работе был рассчитан спектр показателей Ляпунова и отслеживались его изменения при варьировании коэффициента $k_{d}$. Известно, что показатели Ляпунова являются мощным инструментом для анализа эволюции динамических режимов в системах различной природы. Однако при рассмотрении пространственнораспределенных систем, характеризуемых бесконечномерным фазовым пространством, при расчете спектра показателей Ляпунова возникают сложности, связанные с корректным определением состояния рассматриваемой системы и построением набора возмущений [7]. В случае исследуемой в настоящей работе системы задача также осложняется тем, что, наряду с уравнением в частных производных (2), динамика популяции нейронов описывалась при помощи набора ОДУ (1). Для

Письма в ЖТФ, 2017, том 43, вып. 12 
преодоления указанной трудности была разработана модификация методики, предложенная нами ранее для распределенных систем $[8,9]$, а именно, опорное состояние исследуемой системы было задано как

$$
\begin{aligned}
& U(x, y, t)=\left(v_{\overline{1, N}}(x, y, t), w_{\overline{1, N}}(x, y, t), z(x, y, t)\right)^{T}, \\
& v_{\overline{1, N}}(x, y, t)=v_{\overline{1, N}}(t), \\
& w_{\overline{1, N}}(x, y, t)=w_{\overline{1, N}}(t), \quad \forall x, \quad y \in r .
\end{aligned}
$$

На рис. 2 приведены зависимости от времени ляпуновских сумм, нормированных на длину временного ряда, для предельных случаев $k_{d}=1$ (рис. 2,a) и $k_{d}=0$ (рис. 2,b), соответствующих режимам периодической и хаотической динамики, продемонстрированных на рис. 1. Видно, что по истечении интервала времени $T=4000$ безразмерных единиц вычисляемое значение показателя Ляпунова становится практически стационарным и не зависит более от времени и числа итераций процедуры Грамма-Шмидта. При этом режим регулярной пространственно-временной динамики (рис. $1, a$ ), которому соответствуют периодические колебания величины $z(x, y)$ (рис. $1, a)$, характеризуется наличием нулевого старшего показателя Ляпунова. В то же самое время из рис. $2, b$ видно, что для динамического режима, характеризующегося образованием ведущего центра (рис. $1, b)$, существует набор из $N_{\Lambda}=39$ старших положительных показателей Ляпунова (рис. 2,b). С точки зрения показателей Ляпунова данный режим может быть рассмотрен как гиперхаотический, при этом большое число положительных показателей в спектре обусловливается бесконечномерным фазовым пространством рассматриваемой системы.

Для исследования сценария перехода от периодической динамики к гиперхаотической в работе были рассчитаны зависимости пятидесяти ${ }^{1}$ старших показателей Ляпунова от значения управляющего параметра $k_{d}$ (рис. 3). Из приведенной зависимости видно, что в случае, когда $0.58<k_{d}<1.0$, в системе реализуется периодический режим, которому соответствует нулевое значение старшего показателя Ляпунова, а при $0>k_{d}>0.26-$ хаотический, характеризующийся наличием

\footnotetext{
${ }^{1} \mathrm{C}$ учетом бесконечномерного фазового пространства исследуемой системы гиперхаотические режимы могут характеризоваться большим $(N \sim 40)$ количеством положительных показателей Ляпунова.
}

Письма в ЖТФ, 2017, том 43, вып. 12 


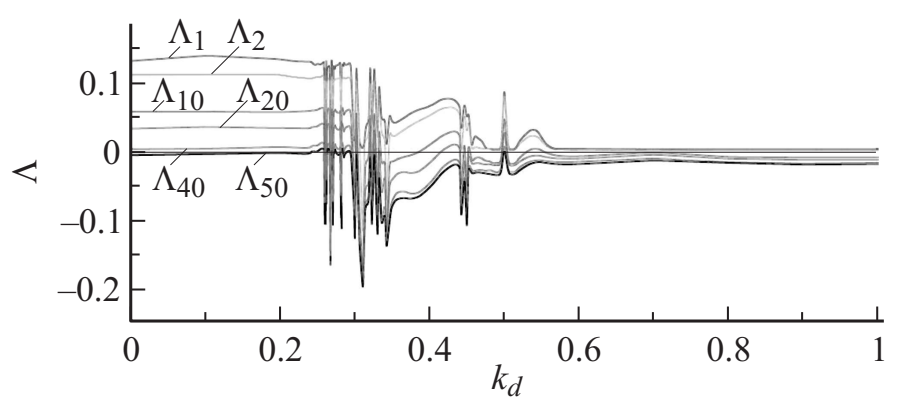

Рис. 3. Значения старших показателей Ляпунова, рассчитанные для различных значений коэффициента $k_{d}$.

$N_{\Lambda}=39$ старших положительных показателей. Следует отметить, что при варьировании значения параметра $k_{d}$ в данных диапазонах усложнения динамики не наблюдается, и в системе реализуются типичные пространственно-временные структуры, приведенные ранее на рис. 1. В то же самое время, при изменении значения управляющего параметра в диапазоне $0.26<k_{d}<0.58$, в системе происходят множественные переходы между регулярными и нерегулярными волновыми режимами.

Подводя итоги, нами был обнаружен и количественно охарактеризован переход между различными типами волновых структур в обобщенной модели нейронной среды. Как было установлено, изменение типа граничных условий в удаленной от ведущего центра (источника волн) области способно кардинальным образом влиять на результирующую пространственно-временную динамику. Применение специальной модификации алгоритма расчета спектра ляпуновских экспонент для случая дискретно-непрерывной активной среды позволило количественно охарактеризовать эволюцию наблюдаемых волновых режимов, и исследовать ее детали. Сообщаемые в данной статье результаты открывают как минимум два перспективных направления дальнейших исследований, одно из которых связано с анализом механизмов генерации самоподдерживающихся структур и автоволн в таких системах, а другое - с дальнейшим развитием методов применения спектра показателей Ляпунова для дискретно-непрерывных активных сред. 
Работа выполнена при поддержке РНФ (грант 14-12-00224). Д.Э. Постнов благодарит Министерство образования и науки РФ (проект 3.1586.2017/ПЧ) за поддержку, воплощенную в части разработки и верификации использованной математической модели. А.Е. Храмов и В.А. Максименко благодарят Министерство образования и науки РФ (проекты 3.861.2017/ПЧ и 3.4593.2017/ВУ) за поддержку, реализованную в части анализа и выделения пространственных паттернов.

\section{Список литературы}

[1] Charles A.C., Baca S.M. // Nat. Rev. Neurol. 2013. V. 9. P. 637.

[2] Tfelt-Hansen P.C. // Cephalalgia. 2010. V. 30. P. 780.

[3] Heiss W.D. // Ann. NY Acad. Sci. 2012. V. 1268. P. 26.

[4] Postnov D.E. et al. // Brain Research. 2012. V. 1434. P. 200.

[5] Postnov D.E. et al. // Phys. Rev. E. 2009. V. 80. P. 031921.

[6] Postnov D.E. et al. // Eur. Phys. J. Special Topics. 2010. V. 187. P. 241.

[7] Купцов П.В. // Известия вузов. ПНД. 2011. Т. 18. № 5. С. 93.

[8] Hramov A.E. et al. // Phys. Plasmas. 2012. V. 19. P. 082302.

[9] Короновский А.А. и др. // Письма в ЖТФ. 2011. Т. 37. В. 13. С. 40.

Письма в ЖТФ, 2017, том 43, вып. 12 